\title{
Enhancement of UV-induced nucleotide excision repair activity upon forskolin treatment is cell growth-dependent
}

\author{
Jeong-Min Lee, Jeong-Min Park \& Tae-Hong Kang ${ }^{*}$ \\ Department of Biological Science, Dong-A University, Busan 49315, Korea
}

Forskolin (FSK), an adenylyl cyclase activator, has recently been shown to enhance nucleotide excision repair (NER) upon UV exposure. However, our study revealed that this effect was detected in human skin epithelial ARPE19 cells only in growing cells, but not in non-cycling cells. When the cells were grown at low density ( $70 \%$ confluence), FSK was capable of stimulating cAMP responsive element binding (CREB) phosphorylation, a marker for FSK-stimulated PKA activation, and resulted in a significant increase of NER activity compared to control treatment. However, cells grown under $100 \%$ confluent conditions showed neither FSK-induced CREB phosphorylation nor the resulting NER enhancement. These findings indicate that cellular growth is critical for FSK-induced NER enhancement and suggest that cellular growth conditions should be considered as a variable while evaluating a reagent's pharmacotherapeutic efficacy. [BMB Reports 2016; 49(10): 566-571]

\section{INTRODUCTION}

Forskolin (FSK) is a general activator of adenylyl cyclase and therefore increases intracellular cyclic AMP (cAMP) levels (1). Through the protein kinase A (PKA)-mediated signaling pathway, FSK treatment triggers the phosphorylation of CAMP responsive element binding protein (CREB) (2). The melanocortin 1 receptor (MC1R), a G-protein-coupled receptor that signals through CAMP and PKA, regulates melanocyteassociated processes including melanocyte growth and differentiation, melanin pigment synthesis, and adaptive tanning (3). Because cAMP is a critical second messenger during melanocyte stimulating hormone (MSH)-induced melanocortin 1 receptor activation, FSK has been developed as a promising topical agent to protect the skin from the side

${ }^{*}$ Corresponding author. Tel: +82-51-200-7261; Fax: +82-51-2007269; E-mail: thkang@dau.ac.kr

https://doi.org/10.5483/BMBRep.2016.49.10.097

Received 13 June 2016, Revised 19 July 2016, Accepted 27 July 2016

Keywords: ATR, Cell growth, Forskolin, Nucleotide excision repair, Ultraviolet (UV) effects of ultraviolet (UV)-induced skin damage $(4,5)$.

UV radiation has been linked to skin cancer because it generates mutagenic DNA photolesions such as cyclobutane pyrimidine dimer (CPD) and pyrimidine (6-4) pyrimidone photoproduct (6-4PP) (6). In humans, nucleotide excision repair (NER) is the sole mechanism for the removal of UVinduced photolesions, and thus genetic defects in NER cause UV-sensitivity syndromes including xeroderma pigmentosum (XP) and Cockayne syndrome (7). The NER is an error-free repair mechanism that requires seven core $\mathrm{XP}$ proteins (XPA-XPG). It begins with the recognition of DNA damage, followed by dual incisions and release of the lesion-bearing DNA strand. The resulting 24- to 32-nucleotide gap is resynthesized using the undamaged complementary strand as a template (8).

The DNA damage-induced cell cycle checkpoint stops cell cycle progression for ensuring sufficient time for DNA repair. ATM (ataxia telangiectasia-mutated) and ATR (ATM and Rad3-related) are two major DNA damage checkpoint kinases in mammals (9). While ATM is a key kinase that orchestrates DNA double-strand break-mediated checkpoint activation, the ATR pathway senses DNA single-strand lesions, including the damage generated by UV irradiation. Previous studies have shown that the ATR-mediated checkpoint pathway is vital to regulate the NER pathway (10-12). For instance, XPA was shown to be directly phosphorylated by ATR at Ser196 in response to UV irradiation, and XPA-deficient cells containing recombinant phospho-deficient XPA protein showed increased sensitivity to UV irradiation compared to that of cells expressing wild-type XPA (13). In addition, a recent study revealed that PKA can phosphorylate ATR at Ser435, which is critical for recruitment of XPA to UV photolesions on DNA, thus accelerating NER activity (14).

In this study, we reassessed the pharmacotherapeutic effect of FSK, an agonist of the PKA pathway during UV-induced DNA damage under different cell growth conditions (i.e., cycling and non-cycling). When the cells were grown at low density $(70 \%$ confluence, at which cells can proliferate), FSK accelerated the removal rate of UV-induced photolesions in genomic DNA through cAMP-mediated PKA activation, as previously reported (14). In contrast, however, when the cells were cultured in $100 \%$ confluent conditions (at which cells do not proliferate), enhanced NER activity driven by FSK

ISSN: 1976-670X (electronic edition)

Copyright (c) 2016 by the The Korean Society for Biochemistry and Molecular Biology

(c) This is an open-access article distributed under the terms of the Creative Commons Attribution Non-Commercial License (http://creativecommons.org/licenses/by-nc/4.0) which permits unrestricted non-commercial use, distribution, and reproduction in any medium, provided the original work is properly cited. 
treatment was not observed. Thus, our results suggest that cellular growth conditions should be considered during assessment of a reagent's pharmacotherapeutic efficacy.

\section{RESULTS}

A previous study had clearly shown that pharmacological activation of the PKA pathway by treatment with the phytochemical FSK can enhance NER activity in human melanocytes during UV-induced DNA damage responses (14). This emphasizes the potential application of FSK as a skinprotecting agent against UV damage. However, the pharmacotherapeutic efficacy of FSK has not yet been tested in different cellular growth conditions (cycling versus non-cycling cells). To address this issue, we compared FSK effects on sub-confluent $(70 \%$; cycling) and confluent $(100 \%$; noncycling) human retinal pigment epithelial ARPE19 cells. To confirm that the cells grown under these conditions were cycling or non-cycling, we measured the level of DNA synthesis using a 5-ethynyl-2'-deoxyuridine (EdU) incorporation assay. As shown in Fig. 1A, after 30 minutes of incubation with EdU in culture medium, approximately $30 \%$ of the sub-confluent cells were labeled with EdU, indicating that cells in this culture condition were undergoing DNA replication (cycling). Conversely, none of the ARPE19 cells maintained at $100 \%$ confluence showed EdU staining, thus hereafter we designated this condition as "non-cycling." In addition, compared to the cycling cells, non-cycling cells showed a significant reduction of both CDT1 and cyclin B1, which are required for cell cycle progression specifically in the G1/S and G2/M phase transitions, respectively (Fig. 1B).

We then analyzed the pharmacological potency of FSK by measuring the level of CREB phosphorylation (p-CREB) (15). In cycling ARPE19 cells, as expected, FSK treatment $(20 \mu \mathrm{M})$ induced 3-fold higher p-CREB levels compared to solvent-only $(0 \mu \mathrm{M})$ control (Fig. 1B \& 1C). In non-cycling cells, however, FSK-mediated p-CREB induction was compromised, even when twice the concentration of FSK $(40 \mu \mathrm{M})$ was applied (Fig. 1B \& 1C). We confirmed that FSK was incapable of PKA activation in the non-cycling condition of different cells including human normal fibroblasts WS1 and Hs895.sk (Fig. 1D). Next, we examined the possibility that non-cycling cells might be unable to internalize the reagent. To test this, we investigated the cell's uptake capability of calcein-acetoxymethyl (calcein-AM), a cell-permeable agent with molecular weight (MW) of $995 \mathrm{~g} / \mathrm{mol}$ whose $\mathrm{MW}$ is twice higher than FSK $(410.5 \mathrm{~g} / \mathrm{mol})$. In live cells, non-fluorescent calcein-AM is converted to green-fluorescent calcein by the action of intracellular esterases (16). As shown in Fig. 1E, both cycling and non-cycling cells showed green fluorescence, indicating an ability to take in calcein-AM and suggesting it is less likely that non-cycling cells would have defective FSK uptake. These results suggest that the compromised $p$-CREB induction in non-cycling cells upon FSK treatment might be due to the rewiring of intracellular signaling pathways in response to contact inhibition of cell growth.

Next, we examined the cellular growth-dependent effects of FSK in response to UV damage. We quantitatively analyzed UV-induced $\gamma-\mathrm{H} 2 \mathrm{AX}$ foci formation using a micropore UV irradiation technique that enabled us to induce localized UV lesions in the nucleus. Cycling or non-cycling ARPE19 cells were exposed to UV radiation through an isopore membrane filter and cultured for various time periods before fixation for immunostaining of $\gamma-\mathrm{H} 2 \mathrm{AX}$, an indicator of DNA breaks. As the recovery time allowed, the number of $\gamma-\mathrm{H} 2 \mathrm{AX}$ foci-positive cells was gradually reduced, in both cycling and non-cycling cells. However, the cycling cells exhibited more efficient removal of DNA breaks upon FSK treatment (Fig. 2A).
A

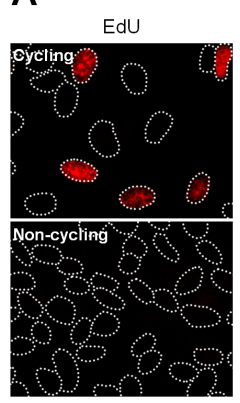

B

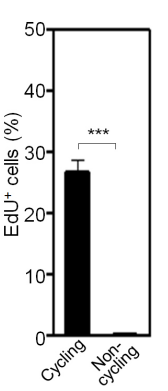

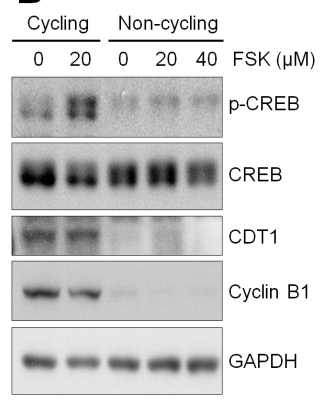

C

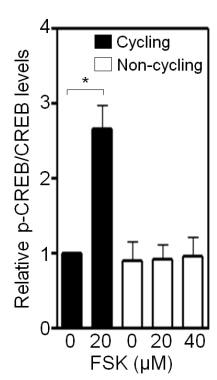

D

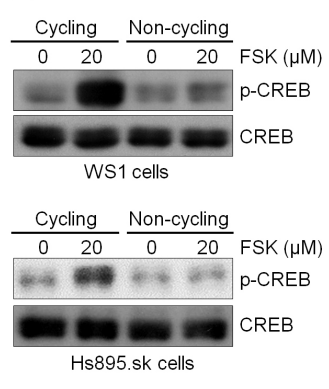

E

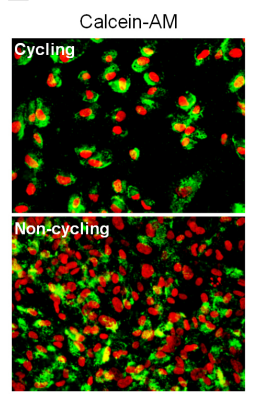

Fig. 1. Cell growth-dependent effect of FSK on CREB phosphorylation. (A) DNA replication in ARPE19 cells was assessed by EdU staining. The cell nuclei were counterstained with Hoechst dye and indicated with dotted lines. The graph represents quantitative analysis of EdU labeling in cycling and non-cycling cells. (B) Protein levels extracted from ARPE19 cells treated with or without of FSK for 1 hour were analyzed by immunoblotting with the indicated antibodies. (C) Quantitative analysis of CREB phosphorylation measured in (B). Bars and error bars represent the mean and SD, respectively, from three independent experiments $(* P<0.05$; $* * * P<0.001)$. (D) Proteins extracted from WS1 and Hs895.sk cells treated with or without of FSK for 1 hour were analyzed by immunoblotting with the indicated antibodies. (E) Calcein-AM uptake analysis in cycling and non-cycling ARPE19 cells. 
A

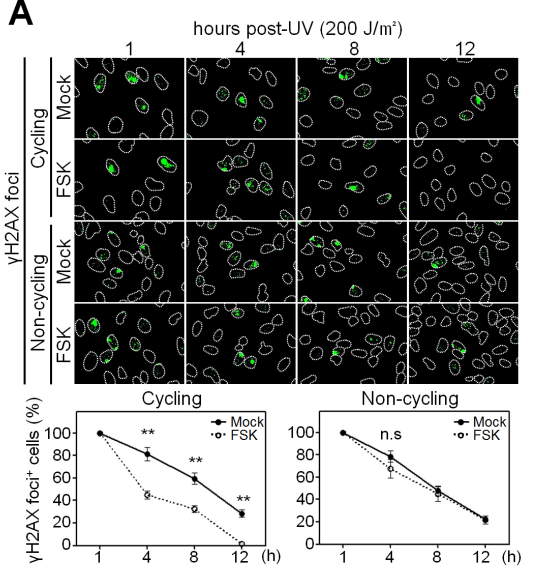

B

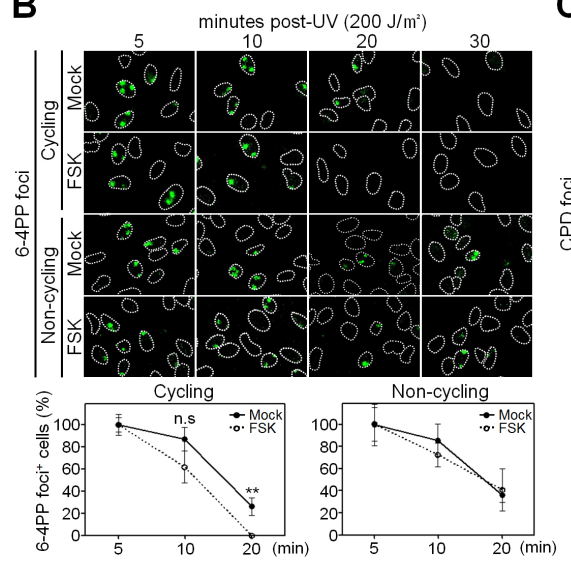

C

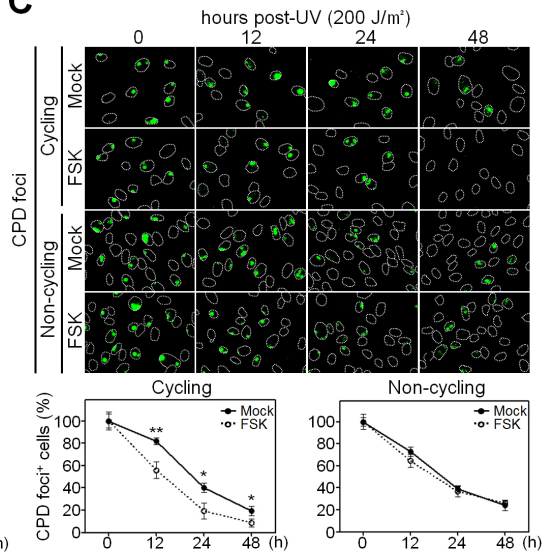

Fig. 2. Cell growth-dependent effect of FSK on the removal of UV-induced photolesions. (A-C) ARPE19 cells were treated with $20 \mu \mathrm{M}$ FSK for 30 minutes before local UV irradiation $\left(200 \mathrm{~J} / \mathrm{m}^{2}\right.$ ) using an isopore filter with $5 \mu \mathrm{m}$ pores, then immunostained for $\gamma$-H2AX (A), 6-4PP $(B)$, and CPD (C), and Hoechst-stained nuclei are indicated with dotted lines. Bars and error bars represent the mean and SD, respectively, from three independent experiments $(\mathrm{ns}=$ no significant difference; $* \mathrm{P}<0.05 ; * * \mathrm{P}<0.01$ ).

Because UV-induced $\gamma$-H2AX formation is associated with NER activity (17), we next measured UV lesion-specific NER rates. $\mathrm{CPD}$ is a non-helix-distorting modification that is the most abundant form of UV-induced photolesion. CPD shows slower repair kinetics when compared to 6-4PP, which is less frequent than CPD but generates a helix-distorting lesion that is more easily recognized and removed by the NER factors (6). Indeed, after $200 \mathrm{~J} / \mathrm{m}^{2}$ of local UV irradiation, most 6-4PP foci were removed within an hour, while removal of some CPD foci required more than 2 days (Fig. $2 B$ \& C). Similar to the results of $\gamma-\mathrm{H} 2 \mathrm{AX}$ removal, FSK-augmented NER activity resulting in 6-4PP and CPD removal was detected only in cycling cells, but not in non-cycling cells (Fig. 2B \& C).

Activation of PKA has been shown to be a prerequisite for FSK-induced enhancement of NER activity (14). Indeed, pretreatment of H89 (a PKA inhibitor) successfully suppressed FSK-induced CREB phosphorylation in cycling cells (Fig. 3A). As a consequence, H89 treatment before UV irradiation reverted FSK-augmented 6-4PP repair kinetics in cycling cells to the level in mock treated cells, whereas no further inhibitory effect of H89 was detected in non-cycling cells (Fig. 3B). Activation of the PKA pathway triggers NER activity by mediating phosphorylation of ATR at Ser435, which enhances XPA recruitment to sites of UV photolesions $(14,18)$. To determine whether FSK's growth-dependent effect on NER activity is regulated by ATR, we treated cells with the ATRspecific inhibitor VE822 before UV irradiation. As shown in Fig. 4A, VE822 suppressed UV-induced CHK1 phosphorylation that is triggered by ATR activation in cycling cells, but showed no effect on ATM-mediated CHK2 phosphorylation. Subsequently, inhibition of ATR pathway during UV-induced DNA damage resulted in delayed repair kinetics of both 6-4PP (Fig. 4B) and CPD (Fig. 4C) compared to those of control cells.

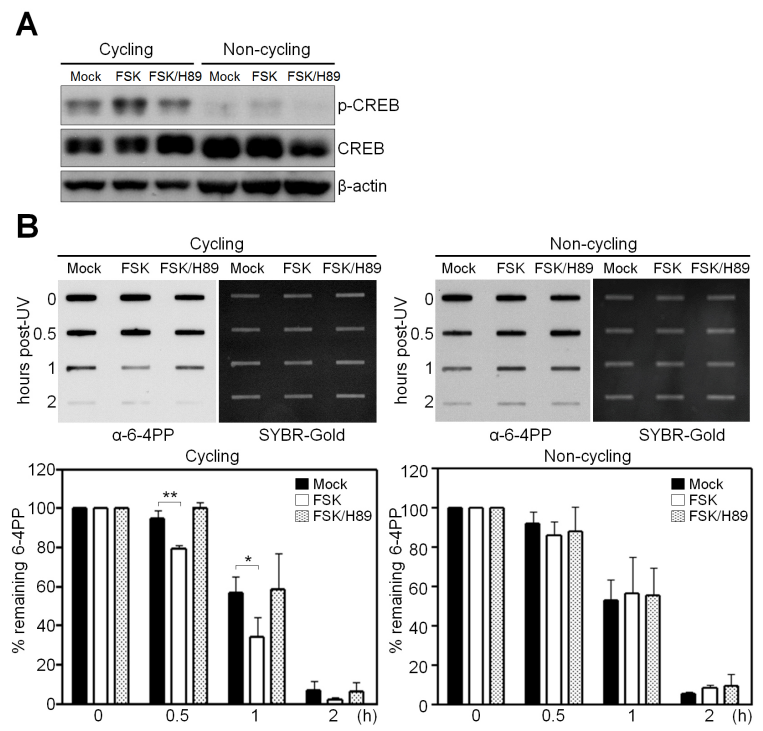

Fig. 3. FSK-mediated NER enhancement during UV-induced DNA damage response is PKA-dependent. (A) Phospho-CREB and CREB protein levels from cells pretreated with FSK or a PKA inhibitor H89 for 1 hour before UV irradiation were analyzed by immunoblotting with the indicated antibodies. Cell lysates were prepared at 2 hours after UV exposure. (B) ARPE19 cells were pretreated with $30 \mu \mathrm{M}$ of a PKA inhibitor $(\mathrm{H} 89)$ for 1 hour before FSK treatment, and irradiated with $20 \mathrm{~J} / \mathrm{m}^{2}$ UV. Cells were allowed to carry out repair for the indicated times, followed by isolation of genomic DNA and immunoslot blotting analysis to detect residual 6-4PP. After immunoslot blotting, the membrane was counterstained with SYBR-Gold to analyze the quantity of genomic DNA loaded. The graphs represent quantitative analysis of NER activity toward 6-4PP lesions. Bars and error bars represent the mean and SD, respectively, from three independent experiments $(* \mathrm{P}<0.05$; $* * \mathrm{P}<0.01)$. 
A

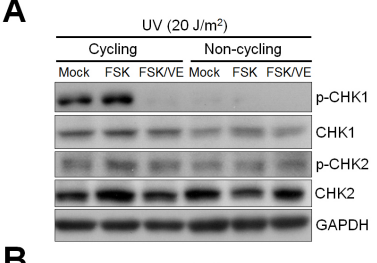

B Cycling
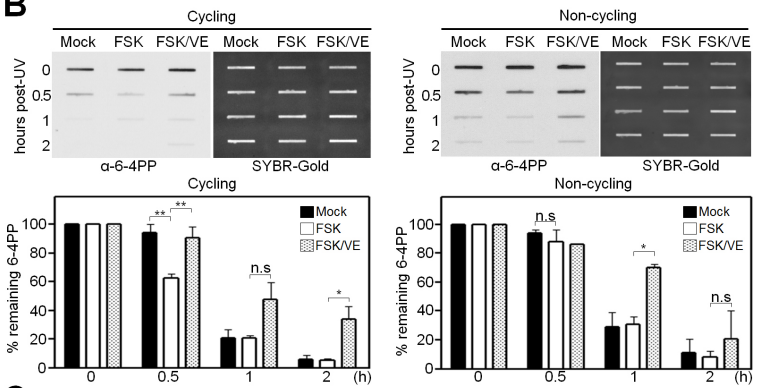

C
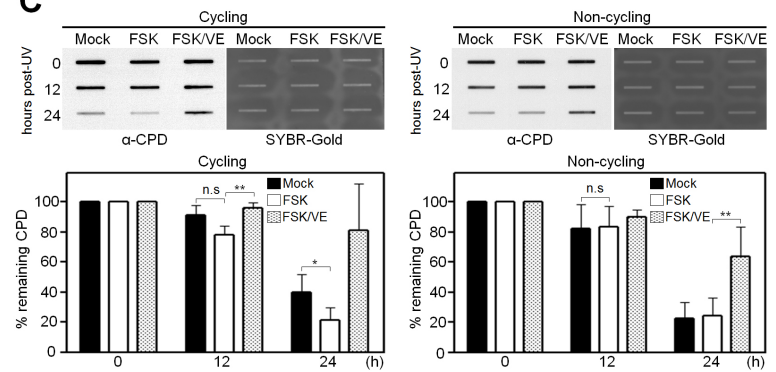

Fig. 4. Pharmacological inhibition of ATR suppresses FSK-potentiated NER activity. (A) Protein levels from cells pretreated with FSK or an ATR inhibitor VE822 for 1 hour before UV irradiation were analyzed by immunoblotting with the indicated antibodies. Cell lysates were prepared at 2 hours after UV exposure. (B, C) Cells pretreated with $5 \mu \mathrm{M}$ of an ATR inhibitor (VE822) for 1 hour before FSK treatment were exposed $20 \mathrm{~J} / \mathrm{m}^{2}$ UV and removal rates of 6-4PP (B) and $\mathrm{CPD}$ (C) lesions were measured using immunoslot blotting. The membrane was counterstained with SYBR-Gold to analyze the quantity of genomic DNA loaded. Bars and error bars represent the mean and SD, respectively, from three independent experiments $\left(\mathrm{ns}=\mathrm{no}\right.$ significant difference; ${ }^{*} \mathrm{P}$ $<0.05$; ** $<$ 0.01).

Taken together, our results confirm that the FSK-PKA-ATR axis regulating NER enhancement during the UV-induced DNA damage response functions in ARPE19 cells as previously reported when the cells are cycling, but this is not true for non-cycling cells.

\section{DISCUSSION}

NER is the sole mechanism for removal of UV-induced photolesions in humans (19). Thus, a reagent that could enhance NER activity during UV irradiation would be a good candidate for development of a skin-protecting agent. Recent reports demonstrated that, in addition to promoting melanin genesis in melanocytes (20), FSK can also directly potentiate
NER activity through the CAMP-PKA-ATR axis in response to UV damage (14). FSK first triggers accumulation of intracellular CAMP, followed by activation of PKA, which phosphorylates ATR, facilitating XPA recruitment to photolesions for accelerated removal by the NER mechanism (14). Our results partially support these previous findings. Indeed, we confirmed that this signaling axis operates in ARPE19 cells, as demonstrated by the ability of FSK to accelerate the removal of UV-induced photolesions. This increased removal was confirmed to occur through the PKA-ATR pathway using specific inhibitors for molecules in this signaling pathway. However, this was observed only in cycling cells but not in non-cycling cells.

To understand the reason for the non-responsiveness of non-cycling cells to FSK, we first addressed our hypothesis that non-cycling cells might be unable to internalize FSK. However, this seemed unlikely to be the case because there was no discrepancy in the ability of cycling and non-cycling cells to internalize calcein-AM (Fig. 1E). Next, by measuring the p-CREB levels upon FSK treatment, we assessed the pharmacological potency of FSK in the two different cell growth conditions. Surprisingly, FSK, a potent adenylyl cyclase agonist, failed to generate CAMP in non-cycling cells. Subsequently, no activation of the PKA-ATR pathway was detected in noncycling cells. Taken together, our data indicate that cell growth status is an important matter to consider during evaluation of pharmacotherapeutic efficacy.

\section{MATERIALS AND METHODS}

\section{Cell culture and FSK treatment}

ARPE19 (human retinal pigment epithelial), human normal skin fibroblast WS1 and Hs895.sk cells were obtained from American Type Culture Collection and cultured in Dulbecco's Modified Eagle's Medium (Hyclone) supplemented with 10\% fetal bovine serum (Hyclone) and 1\% penicillin-streptomycin (Hyclone). Cells were incubated at $37^{\circ} \mathrm{C}$ in a humidified incubator supplemented with $5 \% \mathrm{CO}_{2}$. Cells were treated with FSK (Sigma-Aldrich) for 30 minutes before UV irradiation (20 $\mathrm{J} / \mathrm{m}^{2}$ ). If necessary, cells were treated with 5 or $30 \mu \mathrm{M}$ of specific inhibitors targeting ATR (VE822; Selleck Chemicals) or PKA (H89; Selleck Chemicals) for 1 hour before FSK treatment.

\section{Cell proliferation analysis and calcein-AM uptake assay}

Proliferating cells were detected using the Click-iT EdU Alexa Flour 594 Imaging Kit (Thermo Fisher Scientific). Briefly, cells were incubated with $10 \mu \mathrm{M}$ EdU for 30 minutes, fixed with $4 \%$ formaldehyde for 10 minutes, and treated with $0.5 \%$ Triton X-100 for 20 minutes at room temperature. After washing with PBS, the cells were treated with Click-iT reaction cocktail for 30 minutes and cell nuclei were counterstained with Hoechst 33342 (Sigma-Aldrich) for 20 minutes. The percentage of EdU-positive cells among all Hoechst-stained cells was calculated for quantitative analysis. Internalization of calcein-AM (Enzo Life Sciences) was determined by measuring 
the intensity of green-fluorescent calcein in the cells pretreated with $1 \mu \mathrm{M}$ calcein-AM for 30 minutes. After washing with PBS, cell nuclei were counterstained with Hoechst 33342 (SigmaAldrich) for 5 minutes. The images were captured using a fluorescence microscope (Nikon) equipped with Nikon imaging software NIS-Elements 4.0. For quantification, at least 500 cells per experiment were analyzed from three independent experiments.

\section{Local UV irradiation and immunofluorescence}

Cells were irradiated with UV-C at a dose of $200 \mathrm{~J} / \mathrm{m}^{2}$ through an isopore polycarbonate filter with pores of $5 \mu \mathrm{m}$ in diameter (EMD Millipore). If necessary, cells were treated with $20 \mu \mathrm{M}$ FSK for 30 minutes before local UV irradiation. After incubation for a range of recovery times, cells were fixed in $4 \%$ paraformaldehyde (Sigma-Aldrich) for 10 minutes at room temperature and permeabilized with $0.5 \%$ Triton X-100 (Bio Basic). Antibodies specific for DNA lesions (CPD and 6-4PP) or breaks $(\gamma-\mathrm{H} 2 \mathrm{AX})$ were used for immunostaining. The images were captured using a fluorescence microscope. For quantification, at least 500 cells per experiment were analyzed from three independent experiments.

\section{Immunoblotting}

For immunoblotting, cells were pretreated with $20 \mu \mathrm{M}$ of FSK for 30 minutes before irradiation with UV at a dose of $20 \mathrm{~J} / \mathrm{m}^{2}$. Harvested cells were resuspended in $100 \mu \mathrm{l} 1 \times$ lysis buffer [20 mM Tris- $\mathrm{HCl}$ (pH 6.8), $150 \mathrm{mM} \mathrm{NaCl}, 1 \mathrm{mM}$ EDTA, $1 \mathrm{mM}$ EGTA, $1 \times$ protease inhibitor cocktail, and 10\% Triton X-100] then sonicated (SONICS). Total proteins $(20 \mu \mathrm{g})$ were separated by SDS-polyacrylamide gel electrophoresis using 10\% polyacrylamide gels and transferred to nitrocellulose blotting membranes using electrophoresis chambers (Bio-Rad). Blocked membranes were incubated with antibodies to CDT1 (Bethyl Laboratories), cyclin B1, CHK1 and CHK2 (Santa Cruz Biotechnology), phospho-CREB, CREB, phospho-CHK1, phosphoCHK2 and GAPDH (Cell Signaling Technology).

\section{Immunoslot blotting}

Genomic DNA was obtained using the Exgene $^{\text {TM }}$ Clinic SV mini kit (GeneAll Biotechnology), and $100 \mu \mathrm{g}$ (to detect CPD lesions) or $400 \mu \mathrm{g}$ (to detect 6-4PP lesions) DNA was vacuumtransferred to a nitrocellulose membrane using a BioDot SF Microfiltration apparatus (Bio-Rad). DNA was crosslinked to the membrane by incubation at $80^{\circ} \mathrm{C}$ for 2 hours under vacuum. Monoclonal antibodies that recognize CPD (Kamiya) or 6-4PP (Cosmo Bio) were used to detect lesions in the genomic DNA. After the immunoslot blot assay, the total DNA loaded onto the membrane was visualized and quantified by SYBR-Gold staining (Thermo Fisher Scientific), and these values were used for the loading control.

\section{Statistics}

Statistical significance was determined by the student's t-test.
Data are presented as the mean \pm SD from at least three independent experiments. Differences were considered significant at $\mathrm{P}$ values $<0.05$. All statistical analyses were performed with GraphPad Prism 5.0 software (GraphPad).

\section{ACKNOWLEDGEMENTS}

This work was supported by the Dong-A University research fund.

\section{REFERENCES}

1. Insel PA and Ostrom RS (2003) Forskolin as a tool for examining adenylyl cyclase expression, regulation, and G protein signaling. Cell Mol Neurobiol 23, 305-314

2. Gonzalez GA and Montminy MR (1989) Cyclic AMP stimulates somatostatin gene transcription by phosphorylation of CREB at serine 133. Cell 59,675-680

3. Rodriguez $\mathrm{Cl}$ and Setaluri V (2014) Cyclic AMP (cAMP) signaling in melanocytes and melanoma. Arch Biochem Biophys 563, 22-27

4. D'Orazio JA, Nobuhisa T, Cui R et al (2006) Topical drug rescue strategy and skin protection based on the role of Mc1r in UV-induced tanning. Nature 443, 340-344

5. Passeron T, Namiki T, Passeron HJ, Le Pape $E$ and Hearing VJ (2009) Forskolin protects keratinocytes from UVB-induced apoptosis and increases DNA repair independent of its effects on melanogenesis. J Invest Dermatol 129, 162-166

6. Pfeifer GP and Besaratinia A (2012) UV wavelengthdependent DNA damage and human non-melanoma and melanoma skin cancer. Photochem Photobiol Sci 11, 90-97

7. de Boer J and Hoeijmakers JH (2000) Nucleotide excision repair and human syndromes. Carcinogenesis 21, 453460

8. Kang TH and Sancar A (2009) Circadian regulation of DNA excision repair: implications for chrono-chemotherapy. Cell Cycle 8, 1665-1667

9. Smith J, Tho LM, Xu N and Gillespie DA (2010) The ATM-Chk2 and ATR-Chk1 pathways in DNA damage signaling and cancer. Adv Cancer Res 108, 73-112

10. Lee TH, Park JM, Leem SH and Kang TH (2014) Coordinated regulation of XPA stability by ATR and HERC2 during nucleotide excision repair. Oncogene 33, 19-25

11. Ray A, Milum K, Battu A, Wani G and Wani AA (2013) NER initiation factors, DDB2 and XPC, regulate UV radiation response by recruiting ATR and ATM kinases to DNA damage sites. DNA Repair (Amst) 12, 273-283

12. Park JM, Choi JY, Yi JM et al (2015) NDR1 modulates the UV-induced DNA-damage checkpoint and nucleotide excision repair. Biochem Biophys Res Commun 461, 543-548

13. Wu X, Shell SM, Liu Y and Zou Y (2007) ATR-dependent checkpoint modulates XPA nuclear import in response to UV irradiation. Oncogene 26, 757-764

14. Jarrett SG, Wolf Horrell EM, Christian PA et al (2014) PKA-mediated phosphorylation of ATR promotes recruitment of XPA to UV-induced DNA damage. Mol Cell 54, 
999-1011

15. Misra UK and Pizzo SV (2005) Coordinate regulation of forskolin-induced cellular proliferation in macrophages by protein kinase A/cAMP-response element-binding protein (CREB) and Epac1-Rap1 signaling: effects of silencing CREB gene expression on Akt activation. J Biol Chem 280, 38276-38289

16. Neri S, Mariani E, Meneghetti A, Cattini L and Facchini A (2001) Calcein-acetyoxymethyl cytotoxicity assay: standardization of a method allowing additional analyses on recovered effector cells and supernatants. Clin Diagn Lab Immunol 8, 1131-1135

17. Matsumoto M, Yaginuma K, Igarashi A et al (2007) Perturbed gap-filling synthesis in nucleotide excision repair causes histone $\mathrm{H} 2 \mathrm{AX}$ phosphorylation in human quiescent cells. J Cell Sci 120, 1104-1112

18. Jarrett SG, Wolf Horrell EM, Boulanger MC and D'Orazio JA (2015) Defining the Contribution of MC1R Physiological Ligands to ATR Phosphorylation at Ser435, a Predictor of DNA Repair in Melanocytes. J Invest Dermatol 135, 3086-3095

19. Matsumura Y and Ananthaswamy HN (2004) Toxic effects of ultraviolet radiation on the skin. Toxicol Appl Pharmacol 195, 298-308

20. Newton RA, Cook AL, Roberts DW, Leonard JH and Sturm RA (2007) Post-transcriptional regulation of melanin biosynthetic enzymes by cAMP and resveratrol in human melanocytes. J Invest Dermatol 127, 2216-2227 